\title{
Cognitive-behavioural intervention for self-harm: randomised controlled trial
}

Nadja Slee, Nadia Garnefski, Rien van der Leeden, Ella Arensman and Philip Spinhoven

\section{Background}

Self-harm by young people is occurring with increasing frequency. Conventional in-patient and out-patient treatment has yet to be proved efficacious.

\section{Aims}

To investigate the efficacy of a short cognitive-behavioural therapy intervention with 90 adolescents and adults who had recently engaged in self-harm.

\section{Method}

Participants (aged 15-35 years) were randomly assigned to treatment as usual plus the intervention, or treatment as usual only. Assessments were completed at baseline and at 3 months, 6 months and 9 months follow-up.

\section{Results}

Patients who received cognitive-behavioural therapy in addition to treatment as usual were found to have significantly greater reductions in self-harm, suicidal cognitions and symptoms of depression and anxiety, and significantly greater improvements in self-esteem and problem-solving ability, compared with the control group.

\section{Conclusions}

These findings extend the evidence that a time-limited cognitive-behavioural intervention is effective for patients with recurrent and chronic self-harm.

\section{Declaration of interest}

None. Funding detailed in Acknowledgements.
In recent years there has been a marked rise in the frequency of young people engaging in self-harm. ${ }^{1}$ Identifying those who are at risk is important because every episode of self-harm increases the risk of future episodes ${ }^{2}$ and, eventually, of suicide. ${ }^{3}$ In this paper the term 'self-harm' includes self-poisoning, with or without suicidal intent. ${ }^{4}$ The risk of self-harm increases when multiple risk factors are present. ${ }^{2}$ The presence of a severe psychiatric disorder such as major depression is among the strongest predictors of selfharm. ${ }^{5}$ Anxiety, especially if acute and intense, also has an important role. ${ }^{6}$ In addition to anxiety, patients who self-harm describe feelings of chronic emptiness, alienation and isolation. ${ }^{7}$ In the context of these unpleasant experiences, they report thoughts of hopelessness, ${ }^{8}$ helplessness, ${ }^{9}$ of being a burden to loved ones, ${ }^{10}$ of unlovability and poor distress tolerance, ${ }^{11}$ and of low self-esteem. ${ }^{12}$ Poor problemsolving ability is assumed to interact with suicidal cognitions, increasing the risk of self-harm. ${ }^{13}$

Although in-patient treatment is the standard of care for people who self-harm, it has never been found efficacious in a controlled clinical trial. ${ }^{14}$ Furthermore, controlled cognitivebehavioural therapy (CBT) intervention studies for self-harm are limited and their results are inconsistent. Tyrer et al reported that brief CBT is no more effective than usual care when it comes to preventing repetition of self-harm, ${ }^{15}$ whereas Brown et al reported positive effects of cognitive therapy on suicide attempts, depression and hopelessness. ${ }^{16}$ In addition, several controlled studies have established the efficacy of dialectical behavioural therapy in reducing self-injury in (female) patients with borderline personality disorder. ${ }^{17}$ Schema-focused therapy has also been found to reduce self-harm effectively in patients with borderline personality disorder. ${ }^{18}$ Furthermore, cognitive-behavioural interventions with a problem-solving component seem to have positive effects on self-harm. ${ }^{19}$ These findings are important, given the strong association between acts of self-harm and the risk of suicide described above. In addition, given the association between negative emotions, suicidal cognitions, problem-solving deficits and self-harm, it is important to assess in more detail the impact of treatment on these correlates of self-harm.

In the study reported here the efficacy of a short, manualised cognitive-behavioural intervention for self-harm was investigated.
This intervention was based on a cognitive-behavioural model of maintenance factors of self-harm. ${ }^{20}$ The model assumed that vulnerability to self-harm can be changed by changing suicidal and negative thinking and problem-solving deficits. The intervention aimed to develop cognitive and behavioural skills for coping with situations that trigger self-harm. Considering the wide range of psychiatric, psychological and social problems that patients present with, the intervention was intended to give therapists a clear framework to orient themselves within the therapy. At the same time, the intervention needed to be flexible enough to be of help to a broad range of patients, including those with high risk of repetition of self-harm and high levels of psychiatric comorbidity. The study was designed to determine the short-term and long-term efficacy of the intervention with respect to the rate of repetition of self-harm as well as emotional problems, suicidal cognitions and problem-solving deficits. It was predicted that the rate of self-harm of participants who received CBT in addition to treatment as usual (TAU) would be lower than in patients who received TAU only, and also that participants from the CBT condition would have significantly lower scores for emotional problems (depression and anxiety) and suicidal cognitions, and significantly higher scores for functional cognitions (self-esteem) and behavioural skills (problem-solving ability) following treatment, than participants from the TAU condition.

\section{Method}

\section{Participants}

Patients aged 15-35 years were included in the study if they had recently engaged in self-harm, defined as both deliberate self-poisoning (overdose) and self-injury. ${ }^{4}$ Patients were excluded if they reported a severe psychiatric disorder (e.g. schizophrenia) requiring intensive in-patient treatment (as assessed during the baseline interview with a structured diagnostic interview: the Mini International Neuropsychiatric Interview (MINI)), ${ }^{21}$ were unable to converse in Dutch, had cognitive impairments or lived outside the region of Leiden. 
The study was conducted at the Leiden University Medical Centre and the Rivierduinen mental health centre from March 2003 until April 2006. The initial sample consisted of 222 individuals who had visited the Leiden University Medical Centre or the local mental health centre because of self-harm. Of these 222 people, 32\% $(n=72)$ could not be reached, because the name, address or telephone number they had left was incorrect. Of the $68 \%(n=150)$ who were contacted, $12 \%(n=26)$ declined to participate and $11 \%(n=24)$ were excluded from the study. Reasons for exclusion were the index episode not being an act of self-harm $(n=3)$, being under 15 years old $(n=1)$, being hospitalised for an extended period because of schizophrenia or alcohol or drug misuse $(n=11)$, being unable to converse in Dutch $(n=3)$, having cognitive impairments $(n=2)$ or living abroad $(n=4)$. As a result, 100 individuals ( $45 \%$ of the initial sample) were invited for the baseline interview. The flow of these participants through the study is illustrated in Fig. 1. It shows that 10 people failed to meet the inclusion criteria: they were unable to converse in Dutch $(n=2)$, had cognitive impairments $(n=1)$ or were living outside the Leiden area $(n=5)$. Two more persons did not want to complete the baseline interview. The 90 individuals who entered the study were randomly assigned to 12 CBT sessions in addition to TAU $(n=48)$ or to TAU only $(n=42)$.

Of the 48 people who were randomly assigned to the CBT condition, $8(17 \%)$ left the study prior to the first session (just after the baseline interview), leaving 40 participants to enter treatment. All 40 participants who entered CBT completed the 12 sessions of therapy, as well as the three follow-up assessments. Reasons for leaving the study just after the baseline interview were 'not wanting to be in therapy' $(n=4)$, having started an alternative treatment $(n=1)$, having moved $(n=2)$ or having run away from home $(n=1)$. Of the 42 participants who were randomly assigned to the TAU condition, $5(12 \%)$ did not complete the 3-month follow-up interview, owing to severe psychiatric disorder $(n=2)$, having moved out of the region $(n=1)$, being sentenced to prison $(n=1)$ or reportedly being too busy $(n=1)$. Three more participants did not complete the 6-month follow-up interview, owing to severe psychiatric disorder $(n=1)$, no longer wishing to participate in the project $(n=1)$ or having died by suicide $(n=1)$. One person did not complete the 9-month follow-up interview because she had died by suicide, bringing the total leaving the study to 9 persons (21\%). Participants completing and leaving the study did not differ demographically, on history of self-harm or on any of the outcome variables at baseline.

\section{Procedure}

On visiting one of the participating centres, individuals who had recently engaged in self-harm received a brochure with a complete description of the study. In this brochure it was announced that the person would be contacted by a member of the research team, who would provide further information on the study and would ask if the person were interested in participating. Informed consent was obtained from all participants, and from parents of adolescents below the age of 16 years. After participants had given written consent they were interviewed in their home or at the medical centre. We allowed a week to pass between a person's index episode of self-harm and entering the study, 2 weeks to pass between the index episode and the initial interview, and 3 weeks between the index episode and the first session of CBT. Those who agreed to participate and were found to be eligible for the study were randomly assigned to 12 sessions of CBT in addition to TAU, or to TAU only. All participants were invited for subsequent assessments 3 months, 6 months and 9 months following the baseline interview. Like the baseline interviews, these
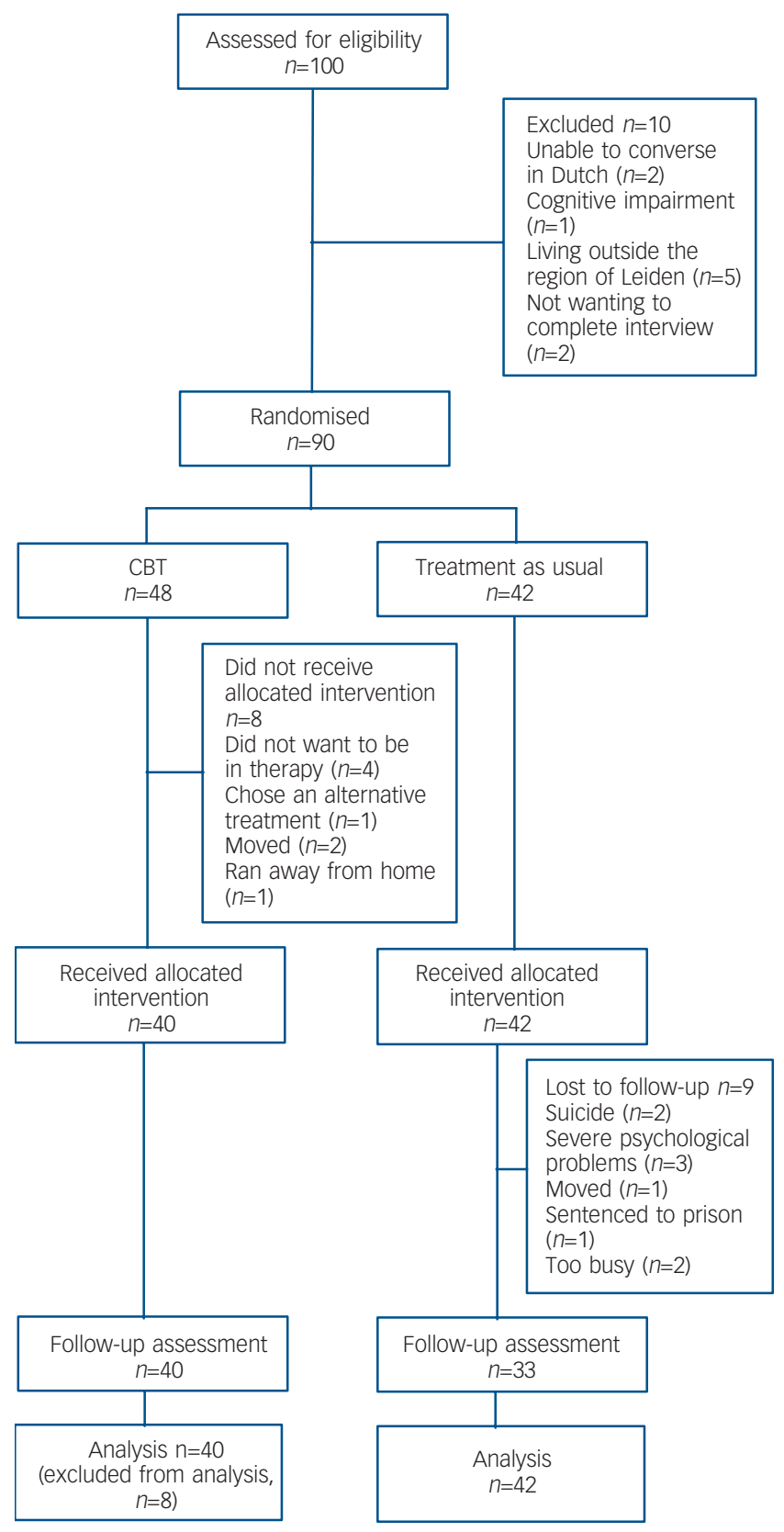

Fig. 1 Flow of participants through the study (CBT, cognitivebehavioural therapy).

interviews were conducted in the participant's home or at the medical centre. The medical ethics committee of the Leiden University Medical Centre approved all procedures.

\section{Design}

Participants were randomly assigned to 12 sessions of CBT in addition to TAU $(n=48)$ or to TAU only $(n=42)$. Randomisation to treatment was accomplished using a computer program and a random-number generator provided by an independent investigator. Stratification was not used. Although masked assessments were conducted at baseline, masking of follow-up assessments was not possible because participants were asked about their use of healthcare services at each assessment. In addition, information regarding treatment assignment was essential to provide care for individuals who were in crisis. The assessments were conducted 
by an independent member of the research team who was not the participant's therapist.

\section{Outcome measures}

The primary outcome measure of the study was the number of episodes of self-harm in the past 3 months, which was assessed using a structured clinical interview. Self-harm was defined as including both deliberate self-poisoning (overdose) and self-injury. ${ }^{4}$ An overdose was defined as the deliberate ingestion of more than the prescribed or recommended amount of a chemical substance with the intention of self-harm. Patients were also asked about incidents of self-injury, which was defined as intentional selfinjury irrespective of the apparent purpose of the act, and included cutting, scratching, punching, kicking and head-banging. In this definition, both the original parasuicide definition of the World Health Organization (WHO)/Euro study and that study's current nomenclature of fatal and non-fatal suicidal behaviour are included, as well as habitual behaviours and self-injuries with no intent to die, which the WHO/Euro study excluded. ${ }^{22,23}$ Thus, all behaviour that was self-initiated with the intent to harm the body (regardless of intent to die) was included.

At each of the four assessments participants were asked about the number of episodes of self-harm in the past 3 months. Other aspects of self-harm were also recorded: suicide intent, motives, the antecedent events and consequences. However, only the number of episodes of self-harm was selected as the primary outcome measure. To investigate the reliability of the assessment of the number of episodes of self-harm, the retrospective self-reports were compared with hospital records, as well as with information coming from the treatment sessions. The correlations between the three measures were high, with correlations ranging from 0.88 to 0.90 . Scores of the number of episodes of self-harm in the past 3 months ranged from 0 to 25 .

Secondary outcome measures assessed by patient self-report at the baseline, 3-month, 6-month and 9-month assessments included depression, anxiety, self-esteem, suicidal cognitions and problem-solving ability.

\section{Depression}

Depression was measured with the Beck Depression Inventory II (BDI-II), ${ }^{24,25}$ a 21-question depression scale with each answer rated $0-3$. Scores range from 0 to 63 . The test has high internal consistency, with $\alpha=0.91 .^{24,26}$ In this study we found a reliability of $\alpha=0.93$.

\section{Anxiety}

Anxiety was measured using a sub-scale of the Symptom Checklist-90, ${ }^{27,28}$ which is a self-report clinical rating scale of psychiatric symptoms. The 'anxiety' sub-scale consists of ten items, assessing whether and to what extent participants reported symptoms of anxiety. Items are measured on a five-point Likert scale, ranging from 'not at all distressing' (0) to 'extremely distressing' (4). Individual sub-scale scores are obtained by summing the ten items (range 0-40). Previous studies have reported alpha coefficients ranging from 0.71 to 0.91 for the anxiety sub-scale. In addition, test-retest reliabilities are found to be good and the sub-scale has been found to show strong convergent validity with other conceptually related scales. ${ }^{28}$ In our study we found a reliability of $\alpha=0.93$ for the anxiety sub-scale.

\section{Self-esteem}

Self-esteem was measured with the Robson Self-Concept Questionnaire, Short version, ${ }^{29}$ an eight-item questionnaire dealing with attitudes and beliefs that people have about themselves, for example, 'I'm glad I am who I am'. All items are self-rated from 1 to 4 ('strongly disagree' to 'strongly agree'). Scores range from 8 to 32 . The scale has good validity and reliability; ${ }^{29}$ in our study we found a reliability of $\alpha=0.82$.

\section{Suicidal cognition}

Suicidal cognitions were measured using the Suicide Cognition Scale. ${ }^{11}$ This comprises 20 questions about core beliefs of perceived burdensomeness ('I am a burden to my family'), helplessness ('no one can help solve my problems'), unlovability ('I am completely unworthy of love') and poor distress tolerance ('when I get this upset, it is unbearable'), with each answer rated 1 (strongly disagree) to 5 (strongly agree). Scores on the total scale range from 20 to 100. Scores on the perceived burdensomeness sub-scale (two items) range from 2 to 10 , scores on the helplessness sub-scale (five items) range from 5 to 25 , scores on the unlovability sub-scale (six items) range from 6 to 30 and scores on the poor distress tolerance sub-scale (seven items) range from 7 to 35 . In this study we found alpha reliability values of 0.96 for the total scale, 0.74 for perceived burdensomeness, 0.88 for helplessness, 0.90 for poor distress tolerance and 0.89 for unlovability.

\section{Problem-solving}

Problem-solving ability was measured with the Coping Inventory for Stressful Situations (CISS) sub-scale task 'oriented coping. ${ }^{30}$ This sub-scale consists of 16 items scored on a five-point Likert scale, referring to the extent to which people make use of problem-solving techniques in the face of stress ('Make an extra effort') with answers ranging from 1 ('not at all') to 5 ('very strongly'. Scores range from 16 to 80 . Across studies, the CISS has proved to be reliable. The internal consistency of the subscales is excellent $(\alpha>0.85) .^{30,31}$ In this study we found a reliability of $\alpha=0.93$.

\section{Other measures}

Demographic information was obtained, as well as information about the use of healthcare services. Baseline characteristics also included suicide intent and motives of the index episode of selfharm. Suicide intent was assessed with the Suicide Intent Scale (SIS), ${ }^{32}$ an instrument with sound psychometric properties. The SIS has 20 items, but only the first 15 items are used for calculating the score. Scoring for each item ranges from 0 to 2 . Items 1-9 are concerned with the act itself, items $10-15$ with the thoughts and feelings associated with the act, and items 16-20 with the respondent's thoughts and feelings about suicide in the present. To assess motives for self-harm, the Reasons for Overdose Scale was used. ${ }^{33}$ The ten motives presented were a subset of those originally developed by Bancroft et al, ${ }^{34}$ including wanting to die, wanting to get relief and wanting to escape. Participants were asked to indicate the extent to which these motives were important to them at the time of the index episode.

\section{Psychiatric diagnosis}

For screening purposes, psychiatric diagnosis was assessed using a short structured diagnostic interview with an administration time of approximately $20-30 \mathrm{~min}$, the MINI. ${ }^{21}$ In this study the Dutch translation of the clinician-rated version of the MINI was used (MINI-CR). ${ }^{35}$ Validation of the MINI-CR against the Structured Clinical Interview DSM-III-R Patient version and the Composite International Diagnostic Interview for ICD-10 showed good to very good kappa values. ${ }^{21}$ 


\section{Intervention}

\section{Cognitive-behavioural therapy}

In addition to usual care (e.g. prescribed psychotropic medication, psychotherapy, psychiatric hospitalisations), participants in the CBT condition received 12 out-patient CBT sessions specifically developed for preventing self-harm. The sessions were provided on a weekly basis or as needed in case of crisis. Ten of the 12 sessions were given weekly; the last two were follow-up sessions. All together, the intervention lasted approximately 5.5 months. The central feature of this intervention was the identification and modification of the mechanisms that maintained self-harm. Thus, the treatment started with the assessment of the most recent episode of self-harm (e.g. circumstances at the time of the episode, motives and reasons for self-harm, cognitions, emotions and behaviour prior to and at the time of the episode). The therapist and patient then investigated how emotional, cognitive and behavioural factors played a part in the maintenance of self-harm. Specific maintenance factors that were addressed included dysfunctional cognitions, emotion regulation difficulties and poor problem-solving. Near the end of therapy relapse prevention was addressed as well. The treatment is first and foremost an individual one. However, involvement of the partner or (non-abusive) parents in the therapeutic process is of great importance, since these patients need the support of others to overcome self-harm. A manual was written to standardise the intervention (available from the authors upon request). To improve treatment compliance, therapists played an active part in keeping patients in treatment (e.g. calling patients to remind them of appointments).

All therapists were experienced practitioners of CBT and accustomed to working with patients who engage in self-harm. Before they took part in the research project, the therapists received 2 days of training in the standardised protocol. To maintain the integrity of treatment, the therapists followed this treatment protocol. In addition, checklists and outlines were used in every session to foster correct execution of the treatment. At monthly meetings the treatment sessions were reviewed and therapists could share their experiences with their colleagues. Issues that were discussed were reactions in the therapist elicited by episodes of self-harm (e.g. sadness, worry, aversion) or problems with treatment compliance. The average number of patients treated by each of the therapists was eight (range seven to nine).

\section{Treatment as usual}

For ethical reasons participants in both study conditions were free to pursue any form of usual treatment they deemed warranted. We recorded three forms of TAU: psychotropic medication, psychotherapy and psychiatric hospitalisations. In addition, we recorded whether psychotherapy in TAU had a focus on self-harm. However, we did not systematically record the specific types of psychotherapy or psychotropic medication that patients in the comparison condition received, nor did we record the specific types of psychotherapy or psychotropic medication those in the experimental condition received in addition to the intervention therapy. As a result, we do not know whether the comparison group and experimental group were equivalent in this respect. Although we did not systematically record specific types of psychotherapy in TAU, most of the interventions involved a limited number (2-30) of sessions of individual psychotherapy such as CBT and interpersonal psychotherapy. Social skills training was also common, especially among adolescents and young adults. No treatment specific to self-harm was reported. These treatments focused instead on specific psychiatric problems (e.g. depression) or on specific needs of the patient (e.g. problems with housing, finances, social isolation).

\section{Statistical procedure}

The study design was constrained to a maximum of four measurements per participant. Given this restriction, a power analysis was performed to determine the sample size needed to detect betweengroup differences on the primary outcome measure - the number of episodes of self-harm. Results obtained with the program PINT $^{36}$ indicated that a sample size of approximately 45 participants in each group would be sufficient to detect a difference in average time slope between the groups of 0.40 (corresponding to a small effect size) with adequate power (0.80) and $\alpha=0.05$. Socio-demographic characteristics and outcome measures of the groups were examined using $t$-tests or chi-squared tests, as appropriate. Multilevel analysis was used to analyse the development of each outcome measure over time; this procedure is especially suited to analysing repeated-measure data because it takes into account the dependencies among observations nested within individuals. Another advantage of this method is its ability to handle missing data, which is also common to the type of longitudinal research discussed in this paper. Random coefficient models were fitted for all outcome measures, allowing for individual variation of intercepts and regression slopes. (In all models time was included as a variable with values $0,3,6$ and 9. Condition, medication use, psychotherapy and suicidal acts were all included as dummy variables: TAU 0 , CBT 1 ; no medication 0 , medication use 1 ; no psychotherapy 0 , psychotherapy 1 ; no suicidal act 0 , suicidal act 1.) Fixed effects of time, condition and the interaction between time and condition were tested using two-tailed $z$-tests. Effects of baseline differences with regard to suicidal acts during the past 3 months on the development of all outcome measures over time were controlled for (suicidal acts condition time). Effects of baseline use of psychotropic medication and psychotherapy on the development of all outcome measures over time were also controlled for: medication use $\times$ condition $\times$ time and psychotherapy $\times$ condition $\times$ time. Models were fitted using MLwiN version 2.02. ${ }^{37}$ In addition, effect sizes were calculated to facilitate comparison of improvement in the CBT condition with improvement in the TAU condition. Effect sizes were derived by calculating the difference of the means on the outcome variables in CBT and TAU at the 3-month, 6-month or 9-month assessment, divided by the pooled standard deviation (see reference 38 for the program to calculate effect sizes). Furthermore, to give an indication of the differences between the conditions for a given variable at baseline, 3-month, 6-month and 9-month assessments, significance levels were calculated using $t$-tests or chi-squared tests.

\section{Results}

Demographic characteristics, psychiatric diagnosis and history of self-harm for participants in the two study groups are reported in Table 1 and online Table DS1. There was no significant difference between the CBT group $(n=40)$ and the TAU group $(n=42)$ on any of the demographic characteristics including gender, age, living situation, marital status, educational level, job status or nationality (Table 1). In addition, no significant difference was found with regard to psychiatric comorbidity, history of previous episodes of self-harm, self-mutilation in the past 3 months or rate of leaving the study (online Table DS1). However, there was a trend towards more suicidal acts in the past 3 months in the TAU group at baseline ( $t=-1.91$, d.f. $=1, n=82 ; P=0.06$ ), and this variable was included in the multilevel analysis models. The baseline table also includes information on the nature of the index episode of self-harm in both groups. The majority of the participants reported self-poisoning at index (CBT 87\%, TAU 91\%; not significant). Suicide intent and motives at index did 
Table 1 Demographic characteristics of participants receiving cognitive-behavioural therapy $(n=40)$ and treatment as usual ( $n=42)$

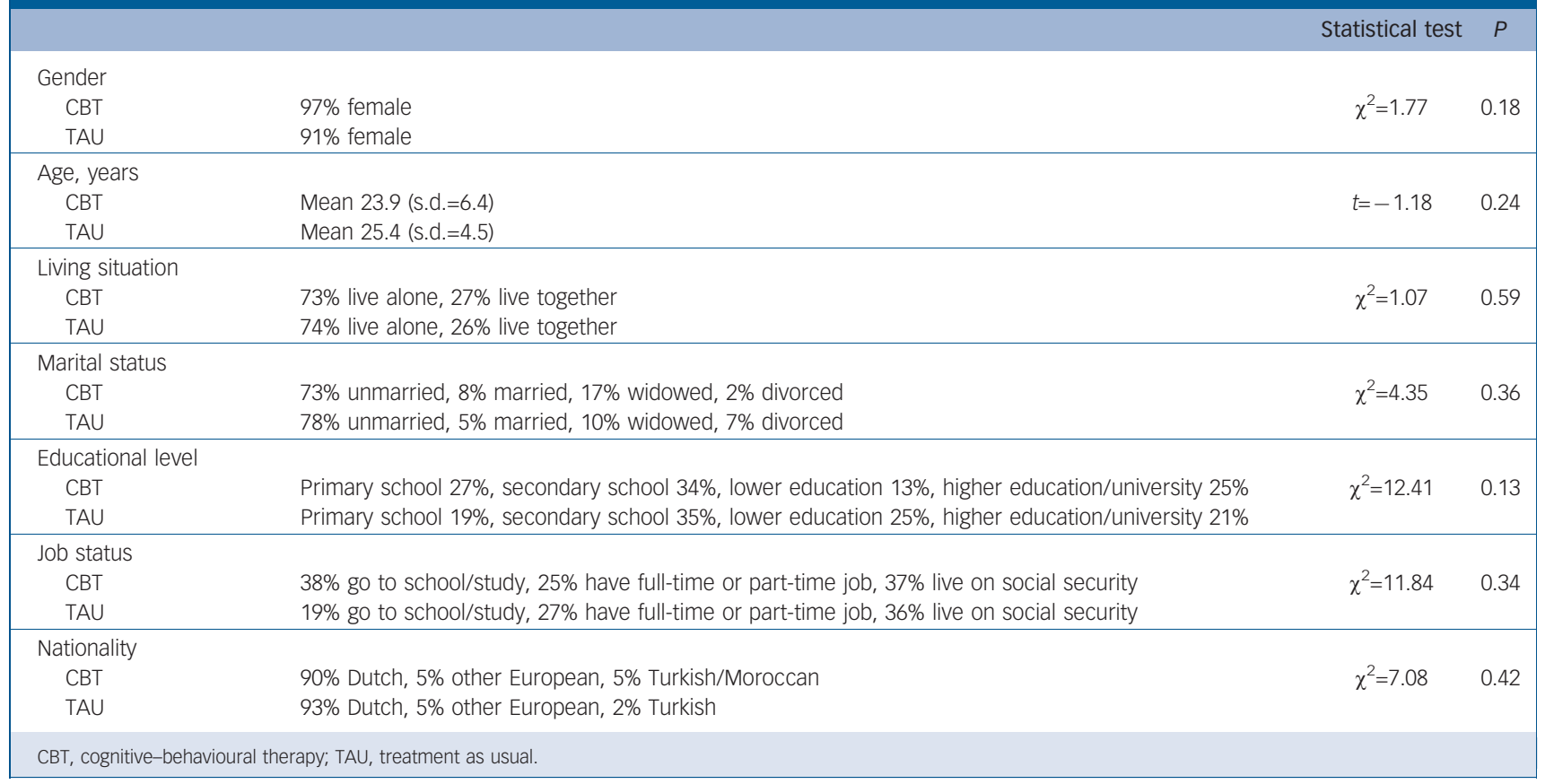

not differ significantly between the groups (online Table DS1). We also investigated whether the groups differed significantly on use of healthcare services: there was no significant baseline difference, except for trends towards more medication use in the TAU group at baseline $\left(\chi^{2}=3.12\right.$, d.f. $\left.=1, n=82 ; P=0.08\right)$ and trends towards more psychotherapy in the TAU group at baseline $\left(\chi^{2}=4.74\right.$, d.f. $=1, n=82 ; P=0.09)$. Both medication use and psychotherapy at baseline were included in the multilevel analysis models. Psychiatric hospitalisation was not included as a covariate in the models because this had not been reported at baseline (Table 2 and online Table DS2). In addition, we used multilevel analysis to determine whether there was any baseline difference between the groups on the primary and secondary outcome measures (see 'condition' effects in Table 3 and online Tables DS3 and DS4). No significant group difference was found on any of these outcome measures (Table 3 and online Tables DS3 and DS4). The mean number of episodes of self-harm during the past 3 months as reported at baseline was 14.42 (s.d.=10.51) in CBT and 11.62 (s.d.=11.42) in TAU; score range 0-25 (Table 2 and online Table DS2).

\section{Primary and secondary outcome measures}

Table 2 shows the means and standard deviation of scores on the primary and secondary outcome measures at baseline and at the 3-month, 6-month and 9-month assessments, including significant group differences based on $t$-tests. In addition, it shows that during the study period two persons in the TAU group died by suicide. The online Table DS2 describes the use of healthcare services by patients in CBT and TAU and group differences based on chi-squared tests.

\section{Outcome of treatment at 9 months}

In Table 3 (multilevel analysis without the 8 early withdrawals, $n=82$ ), online Table DS3 (multilevel analysis with those completing the intervention, $n=73$ ) and online Table DS4 (multilevel analysis with intent-to-treat sample, last observation carried forward, $n=90$ ), fixed effects and corresponding standard errors are reported for the multilevel analysis models regarding the primary and secondary outcome measures. (In multilevel analysis the most commonly used estimation method is maximum likelihood. For this method it is necessary to assume normality for the dependent variable. The distribution of self-harm does not satisfy the normality assumption. However, all conclusions are based on the interpretation of fixed effects. Simulation results show that with the sample size in this study estimates of fixed effects and their standard errors are not seriously affected by non-normality of the residuals. ${ }^{39,40}$ Moreover, a comparison between the maximum likelihood standard errors and the so-called 'robust standard errors', used as a tool to assess model mis-specifications, showed that results for self-harm can be considered reliable and can be interpreted correctly.) The effect of 'time' indicates the overall increase or decrease for each of the outcome measures. The effect of 'condition' indicates the difference between CBT and TAU at baseline. The interaction effect (time $\times$ condition) indicates whether there is a significant difference between CBT and TAU upon the development of the outcome measures over time. To control for baseline differences in suicidal acts during the past 3 months, the interaction of suicidal acts $\times$ condition $\times$ time was included in the models as well. In addition, to control for baseline differences in psychotropic medication use and psychotherapy, the interactions of medication use $\times$ condition time and of psychotherapy $\times$ condition time were included in the models. Fixed effects were tested by two-tailed $z$-tests. Variance components are omitted here because they are not the primary focus of our study.

Multilevel analysis results without the 8 participants who left the study early ( $n=82$; Table 3 ) showed that overall self-harm, depression and suicidal cognitions (total scale and the sub-scales perceived burdensomeness, poor distress tolerance and unlovability) significantly decreased over time. Self-esteem was shown to increase significantly over time. No significant effect was found for 'condition', indicating that on average there was no significant baseline difference between the groups on all outcome measures. The fixed effects of time $\times$ condition showed that there was a significant effect of condition upon the development (increase or decrease) of all outcome measures over time. For instance, 


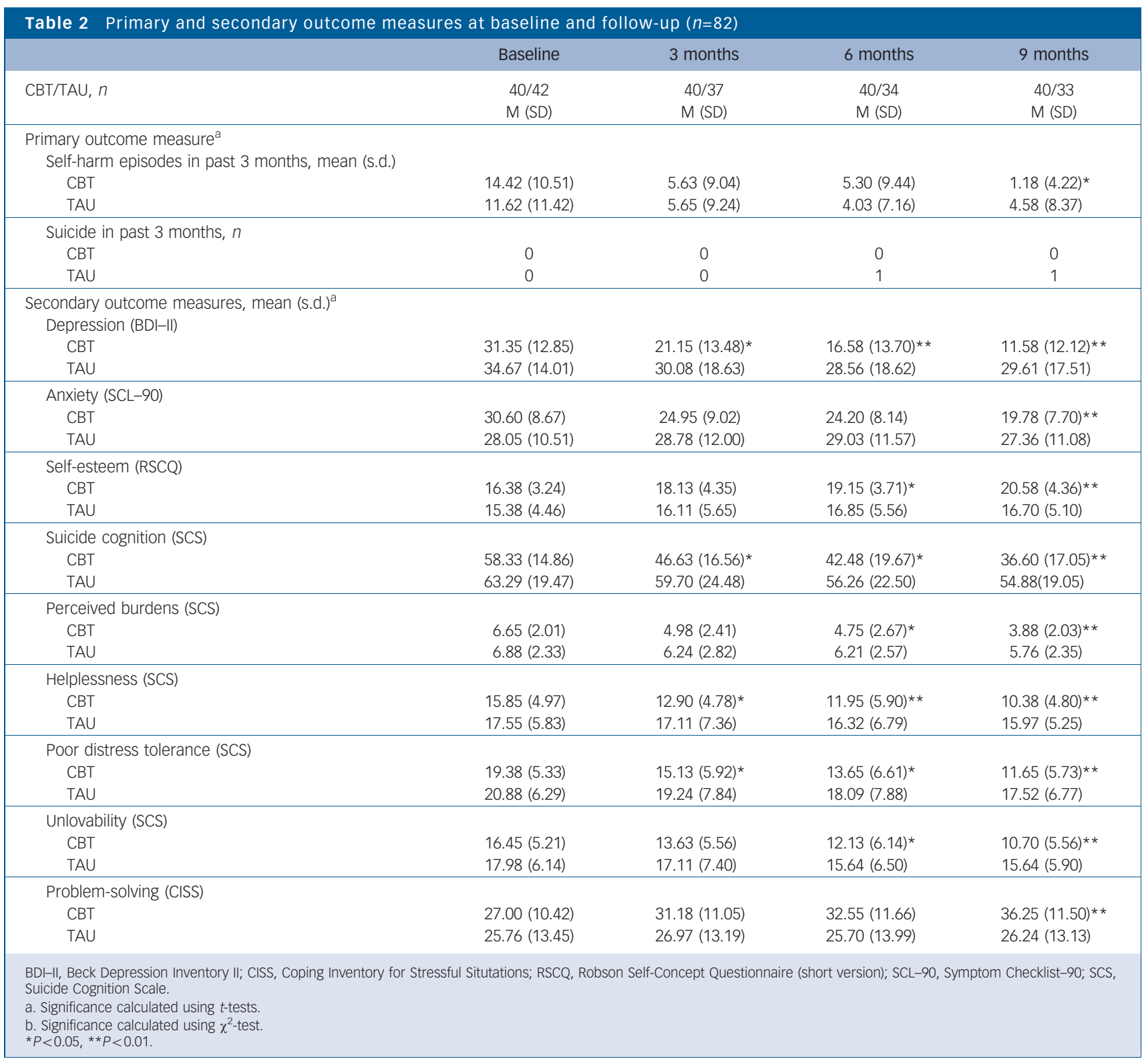

the estimated value of -0.576 in the model for self-harm indicates that the individuals in the CBT group, on average, showed a significant difference in decrease of self-harm compared with individuals in the TAU group (Table 3). The significant time $\times$ condition effects remain with different ways of handling study withdrawals. Multilevel analysis results using the 'completers' sample ( $n=73$; online Table DS3) and the last observation carried forward (LOCF) method ( $n=90$; online Table DS4) showed a slightly smaller effect for 'time' on average, but a similar significant effect for time $\times$ condition as multilevel analysis without the 8 early withdrawals ( $n=82$, see Table 3 ). The most conservative analysis (LOCF) showed the strongest effect for CBT on self-harm $(t=-2.843)$, whereas the more optimistic analysis without the 8 early withdrawals showed the weakest effect for CBT on self-harm $(t=-2.390)$. However, each of the three analyses led to the same significant effect of time $\times$ condition. Moreover, none of the effects of suicidal acts during the past 3 months $\times$ condition $\times$ time were significant, indicating that there was no effect of suicidal acts at baseline on the development of the outcome measures over time in the two conditions. In addition, none of the effects of medication use $\times$ condition $\times$ time was significant, indicating that there was no effect of medication use at baseline on the development of the outcome measures over time in the two conditions. Furthermore, none of the effects of psychotherapy $\times$ condition $\times$ time was significant, indicating that there was no effect of psychotherapy at baseline on the development of the outcome measures over time in the two conditions. These interaction effects are omitted from the models. Table 3 (multilevel analysis without the 8 early withdrawals, $n=82$ ), online Table DS3 (multilevel analysis with completers, $n=73$ ) and online Table DS4 (multilevel analysis with LOCF, $n=90$ ) present the results of a simple model with only three fixed effects, which is preferable because it is easier to understand. In addition, effect sizes were calculated to facilitate comparison of improvement in the CBT condition with improvement in the TAU condition (Table 3 and online Tables DS3 and DS4). Effect sizes of 0.20 indicate small effects, effect sizes of 0.50 indicate medium effects, whereas values of 0.80 indicate large effects. ${ }^{41}$ As can be derived from Table 3 and online Tables DS3 and DS4, the effect sizes become larger during the follow-up period and at the 9-month assessment effect sizes are medium for the difference in self-harm and large to very large for the differences on all other primary and secondary outcome 


\begin{tabular}{|c|c|c|c|c|c|c|}
\hline Variables & $\begin{array}{l}\text { Time } \\
\text { B (s.e.) }\end{array}$ & $\begin{array}{l}\text { Condition }^{\text {a }} \\
\text { B (s.e.) }\end{array}$ & $\begin{array}{c}\text { Time } \times \text { condition } \\
\text { B (s.e.) }\end{array}$ & $\begin{array}{c}\text { Cohen's d } \\
3 \text { months } \\
\text { (post-treatment) } \\
n=40 / 37\end{array}$ & $\begin{array}{l}\text { Cohen's d } \\
6 \text { months } \\
\text { (follow-up) } \\
n=40 / 34\end{array}$ & $\begin{array}{c}\text { Cohen's d } \\
9 \text { months } \\
\text { (follow-up) } \\
n=40 / 33\end{array}$ \\
\hline Self-harm & $-0.794(0.177)^{*}$ & $2.892(2.089)$ & $-0.576(0.241)^{*}$ & 0.0 & 0.06 & 0.53 \\
\hline Depression (BDI-II) & $-0.515(0.223)^{*}$ & $-4.047(2.956)$ & $-1.617(0.302)^{*}$ & 0.56 & 0.75 & 1.24 \\
\hline Anxiety (SCL-90) & $-0.065(0.174)$ & $-0.748(2.018)$ & $-1.038(0.235)^{\star}$ & 0.37 & 0.22 & 0.82 \\
\hline Suicide cognitions total (SCS) & $-0.835(0.304)^{*}$ & $-6.339(3.661)$ & $-1.497(0.411)^{*}$ & 0.64 & 0.67 & 1.03 \\
\hline Perceived burdensomeness (SCS) & $-0.106(0.039)^{\star}$ & $-0.489(0.465)$ & $-0.175(0.052)^{*}$ & 0.49 & 0.56 & 0.88 \\
\hline Helplessness (SCS) & $-0.142(0.089)$ & $-1.987(1.120)$ & $-0.457(0.132)^{*}$ & 0.69 & 0.70 & 1.13 \\
\hline Poor distress tolerance (SCS) & $-0.328(0.106)^{*}$ & $-1.797(1.248)$ & $-0.514(0.143)^{\star}$ & 0.60 & 0.62 & 0.96 \\
\hline Unlovability (SCS) & $-0.268(0.097)^{\star}$ & $-2.074(1.168)$ & $-0.349(0.130)^{*}$ & 0.54 & 0.56 & 0.88 \\
\hline Self-esteem (RSCQ) & $0.141(0.066)^{*}$ & $0.852(0.810)$ & $0.309(0.089)^{*}$ & 0.41 & 0.54 & 0.83 \\
\hline
\end{tabular}

measures between both treatment conditions. The use of parametric statistics with skewed data (the number of episodes of self-harm) might have reduced the effect size estimates as presented in Table 3 and online Tables DS3 and DS4.

Visual inspection of means in Table 2 and effect sizes in Table 3 and online Tables DS3 and DS4 suggests a curvilinear time trend for self-harm in TAU but a linear trend for self-harm in CBT. However, the curvilinear trend did not prove to be significant. We therefore used a linear model for self-harm in CBT as well as TAU.

\section{Use of healthcare services during the study period}

Chi-squared tests were used to examine differences in the use of healthcare services during the study period. Table 2 shows that at baseline, $43 \%(n=17)$ of the patients in the CBT group received psychotherapy. About half of these patients preferred to interrupt their regular psychotherapy schedule for a period of 3 months, which was the period in which 10 out of 12 CBT sessions took place. Between baseline and the 3-month assessment, only $21 \%$ of the patients received CBT for self-harm and regular psychotherapy at the same time. Between the 3-month and 6-month assessments, $28 \%$ of the patients received CBT for self-harm in addition to usual care (Table 2). After the CBT intervention, $52 \%(n=25)$ of the patients continued or began regular psychotherapy. Table 2 also shows that individuals receiving CBT used significantly less psychotropic medication between the baseline and 3 -month assessments $\left(\chi^{2}=3.970\right.$, d.f. $=1, n=77$; $P=0.046)$ and between the 3-month and 6-month assessments $\left(\chi^{2}=4.270\right.$, d.f. $\left.=1, n=74 ; P=0.039\right)$, but not between the 6-month and 9-month assessments. From baseline to the 6-month assessment the number of psychiatric hospitalisations was lower in the CBT group $(n=4)$ than in the TAU group $(n=13)$, but this difference was not significant. However, between the 6-month and 9-month assessments significantly fewer people had been hospitalised in the CBT group compared with the TAU group ( $n=1 v$. $n=7 ; \chi^{2}=6.488$, d.f. $=1, n=73 ; P=0.011$ ).

\section{Discussion}

\section{Main findings}

The short cognitive-behavioural intervention reported here was designed to supplement usual care following an episode of self-harm. Our main study hypothesis, that CBT in addition to
TAU would be more effective in reducing repetition of self-harm than TAU alone, was supported. Furthermore, those who received CBT in addition to TAU were shown to have significantly greater reductions in depression, anxiety and suicidal cognitions, and significantly greater improvements in self-esteem and problemsolving ability. It is reasonable to assume that these positive findings are attributable to the effect of the CBT, given the random assignment as well as the absence of between-group differences with respect to demographic factors, number of episodes of selfharm, history of self-harm, psychopathology and use of healthcare services.

While this study confirms prior studies showing that self-harm can be effectively treated by $\mathrm{CBT},{ }^{17,18}$ it is among the first to suggest that these changes can occur with a brief intervention. Furthermore, this study's findings that time-limited CBT decreases self-harm contrasts with the results reported by Tyrer et al. ${ }^{15}$ The difference in outcome could be attributed to the fact that in our study all participants who began CBT completed all sessions, a fact that probably contributed to its efficacy. The positive treatment effect on self-harm is important given the high suicide risk following self-harm. ${ }^{3}$ The suicidal process appears to have been at least partially deflected by the CBT intervention. ${ }^{42}$ It is especially important that these results are found for people with recurrent and chronic self-harm, with a high risk of repetition and high levels of psychiatric comorbidity.

\section{Possible mechanisms: suicidal cognitions and behavioural skills deficits}

The theoretical model underlying the cognitive-behavioural intervention suggested that vulnerability to self-harm was related to underlying suicidal cognitions and behavioural skills deficits. ${ }^{20}$ From this perspective, reduction in repetition of self-harm following CBT might be seen as a consequence of the therapy reducing specific suicidal thoughts and problem-solving deficits and increasing self-esteem. As expected, over the course of treatment there were marked changes in suicidal cognitions as well as in self-esteem. The significant decrease in suicidal cognitions is especially important, since these are considered to be the main triggers of self-harm, especially for individuals with recurrent and chronic self-harm. ${ }^{11}$ Given the central role of suicidal cognitions in repetition of self-harm, the CBT aimed to increase the patient's 
hope by systematically targeting cognitions of perceived burdensomeness, helplessness, poor distress tolerance and unlovability. This occurred as the therapist, while validating the patient's emotions, modelled hopefulness and the ability to improve the current situation through the identification and modification of unhelpful thoughts as well through the use of effective problemsolving skills.

Helping patients with their current problems was another important element of treatment, because previous research had shown that people who self-harm display poor problem-solving ability, which seems to be independent of mood, ${ }^{12}$ and relatively stable unless intervened upon. ${ }^{43}$ At the start of therapy patients commonly reported feeling overwhelmed by the problems they were facing, believing they were lacking effective problem-solving strategies. By identifying effective strategies that they already used, the idea that control was already part of their repertoire was introduced. In addition, patients were encouraged to develop and use new strategies. ${ }^{44}$ As expected, patients' problem-solving skills significantly improved during treatment.

It is remarkable that effects on secondary measures - particularly depression, suicidal cognitions and problem-solving - were stronger than on the target variable 'self-harm' (the number of episodes of self-harm in the past 3 months). Moreover, changes in these factors seemed to precede changes in self-harm. This suggests that the CBT primarily targeted depression, suicidal cognitions and problem-solving and that the specific self-harm effect (which was only apparent at the 9-month assessment) was a secondary effect. This would be consistent with the assumption that repetition of self-harm could be reduced by revising its maintenance factors. ${ }^{20}$ Given the emphasis in CBT on challenging suicidal thinking and increasing problem-solving ability, either of these aspects might be the mechanisms of change.

\section{Unique additional effect of CBT}

The trajectory for the average patient in the CBT group showed a relatively rapid reduction on many outcome measures early in therapy and this reduction continued as therapy progressed and during the follow-up period. However, this rapid reduction is also found in the TAU group, as indicated by the strong effect of time in the multilevel analysis. This effect might be explained by the fact that people were in crisis when they entered the study; at that time their risk of self-harm was elevated, mood was particularly low, suicidal cognitions were persistent, and these people had great difficulty solving the problems they were facing. It has been argued that crises are by themselves time-limited, even in those exhibiting recurrent and chronic self-harm. ${ }^{11}$ However, the magnitude of the interaction effects in the multilevel analysis clearly show the unique additional effect of CBT. Furthermore, cognitions of helplessness and problem-solving ability did not seem to change significantly over time in the TAU condition. Therefore, it seems that CBT emphasises these important risk factors and possible mediators of treatment effect. Targeting both cognitions of helplessness and problem-solving difficulties might have made the intervention especially effective.

\section{Limitations}

Despite these generally positive findings, several limitations of this study deserve comment.

\section{Assessment of self-harm}

A primary limitation is that the instrument used to assess the number of episodes of self-harm during the preceding 3 months does not have well-established psychometric properties. Validated instruments that cover the number of episodes of self-harm as well as other aspects of self-harm (e.g. circumstances of the act, motives, intent, consequences) were not available at the start of the project.

\section{Duration of the follow-up period}

A second limitation is the absence of an extended follow-up period; a longer period could clarify whether the positive treatment effects persist or even develop further over time. A follow-up period of 12 months would be advisable, because the risk of repetition of self-harm (and completed suicide) is at its greatest during the first 12 months following an episode of self-harm. ${ }^{45}$

\section{Usual treatment}

A third limitation is the way in which usual treatment was specified. We recorded three forms of such treatment: psychotropic medication, psychotherapy and psychiatric hospitalisations. We can conclude that both conditions received a comparable level of care within these general categories of treatment. However, we did not record specific types of psychotherapy or psychotropic medication in the TAU condition. Therefore, it is unclear if the conditions were equivalent in this respect. Future studies should record specific types of usual treatment received by people in the experimental and comparison conditions. Furthermore, the effect of CBT was only demonstrated in relation to TAU. Therefore, it is unclear whether the treatment effects are attributable to specific ingredients of the therapy or to the total package of CBT in addition to TAU. Moreover, since people in the TAU group did not always receive psychotherapy, it is conceivable that the treatment effects in the CBT group were non-specific. In addition, more than half of the patients in the CBT group continued with psychotherapy or began psychotherapy after the self-harm intervention had ended. Future research is necessary to identify variables that mediate treatment effects (e.g. specific cognitions) and to detect the active ingredients of the intervention therapy (e.g. identifying and modifying suicidal cognitions). ${ }^{46}$

\section{Recruitment and withdrawal rates}

A fourth limitation is the low recruitment rate (45\%) and the relatively high rate of withdrawal from the CBT condition (17\%), in which 8 patients left prior to treatment. However, session attendance of those who began the CBT was high. Furthermore, other studies also report that adherence to treatment is a well-known problem among self-harm patients, because of the severity of their psychological distress and the wide range of problems they face (e.g. social, financial, housing) ${ }^{47}$ Importantly, the recruitment and withdrawal rates in this study did not exceed rates reported in similar studies.

\section{Treatment integrity}

A fifth limitation concerns treatment integrity in the CBT condition. This could have been more systematically assessed by rating audiotapes or videotapes of the treatment sessions. Furthermore, the assessments were not carried out masked to treatment group, which might have influenced outcome.

\section{Handling of study withdrawals}

A sixth limitation is the way study withdrawals were handled. Multilevel analysis uses all available data but assumes that withdrawals occur at random, which is questionable in this population. As the assumption of randomness is unlikely, we also executed and reported the results with missing data estimated 
with the LOCF method $(n=90)$. Both this analysis $(n=90)$ and the analysis without the 8 withdrawals $(n=82)$ led to a significant effect of time $\times$ condition. A significant effect of CBT for self-harm was also observed in the 'completers' sample $(n=73)$. The true effect of the CBT is probably somewhere between the conservative LOCF method $(n=90)$ and the more optimistic analysis without the 8 withdrawals $(n=82)$. Remarkably, the LOCF method showed the strongest effect of CBT for self-harm, which is contrary to our expectations.

\section{Personality disorders}

A further limitation is that the presence of personality disorders was not assessed with a structured clinical interview. The presence of borderline personality disorder or a range of personality disorders is likely to have an impact on treatment outcome. A previous study showed that personality disturbance has an impact on repetition of self-harm, patients with borderline personality disorder being most likely to repeat episodes quickly. ${ }^{48}$ Furthermore, with the absence of data on personality disorders, it remains unclear whether the study population has similar Axis II diagnoses to the patients described in the studies of Tyrer et al and Linehan et al. ${ }^{15,17}$ However, the participants in our study strongly endorsed maladaptive beliefs associated with personality disorders, especially borderline and avoidant beliefs (further information available from the authors), to a greater extent than even patients with borderline personality disorder, ${ }^{49}$ which can be seen as an indication of the presence of personality disturbance. The high rate of repetition of self-harm found in this study seems to confirm this. A last limitation is that this study primarily involved young women with Dutch nationality. This absence of diversity limits the generalisability of our findings.

\section{Clinical implications}

In sum, our findings extend the evidence that CBT is effective in patients with chronic and recurrent self-harm. ${ }^{16,17}$ The results of our study are strengthened by the consistency of the results across several outcomes. Adding this short cognitive-behavioural intervention to usual care might provide us with an important tool to prevent repetition of self-harm in people who are at risk. It might, for instance, be the first intervention in a stepped-care programme, in which self-harm is addressed first, followed by a treatment focusing on underlying personality characteristics, such as schema-focused therapy or mentalisation-based treatment. ${ }^{50,51}$ Replication of these findings using a longer follow-up period and more insight into the underlying mechanisms of change is necessary.

\footnotetext{
Nadja Slee, MSC, Nadia Garnefski, PhD, Unit of Clinical Psychology, Rien van der Leeden, PhD, Unit of Methods and Statistics, Leiden University Institute for Psychological Research, Leiden, The Netherlands; Ella Arensman, PhD, Nationa Suicide Research Foundation, Cork, Ireland; Philip Spinhoven, PhD, Unit of Clinica Psychology, Leiden University Institute for Psychological Research, and Department of Psychiatry, Leiden University Medical Centre, Leiden, The Netherlands

Correspondence: Nadja Slee, Department of Clinical, Health and Neuropsychology, Wassenaarseweg 52, PO Box 9555, 2300 RB Leiden, The Netherlands. Email: nadja.slee@planet.nl

First received 5 Mar 2007, final revision 7 Sep 2007, accepted 30 Oct 2007
}

\section{Acknowledgements}

Support for the study was provided by The Netherlands Organisation for Health Research and Development (ZonMw) (contract grant number: 2100.0068). We thank all patients for their participation in the study, as well as the therapists for their careful and thoughtful delivery of the intervention therapy. In addition, we would like to thank the anonymous reviewers for their valuable comments.

\section{References}

1 Evans E, Hawton K, Rodham K, Deeks J. The prevalence of suicide phenomena in adolescents: a systematic review of population-based studies. Suicide Life Threat Behav 2005; 35: 239-50.

2 Colman I, Newman SC, Schopflocher D, Bland RC, Dyck RJ. A multivariate study of predictors of repeat parasuicide. Acta Psychiatr Scand 2004; 109 306-12.

3 Zahl DL, Hawton K. Repetition of deliberate self-harm and subsequent suicide risk: Iong-term follow-up study of 11583 patients. Br J Psychiatry 2004; 185: 70-5.

4 Hawton K, Zahl D, Weatherall R. Suicide following deliberate self-harm: longterm follow-up of patients who presented to a general hospital. $\mathrm{Br} J$ Psychiatry 2003; 182: 537-42.

5 Fawcett J, Clark DC, Busch KA. Assessing and treating the patient at risk for suicide. Psychiatr Ann 1993; 23: 245-55.

6 Busch KA, Clark DC, Fawcett J, Kravitz HM. Clinical features of inpatient suicide. Psychiatr Ann 1993; 23: 256-62.

7 Orbach I, Mikulincer M, Gilboa-Schechtman E, Sirota P. Mental pain and its relationship to suicidality and life meaning. Suicide Life Threat Behav 2003: 33: 231-41.

8 Glanz LM, Haas GL, Sweeney JA. Assessment of hopelessness in suicidal patients. Clin Psychol Rev 1995; 15: 49-64.

9 D'Zurilla TJ, Chang EC, Nottingham EJ, Faccini L. Social problem-solving deficits and hopelessness, depression, and suicidal risk in college students and psychiatric inpatients. J Clin Psychol 1998; 54: 1091-107.

10 Brown SL, vinokur AD. The interplay among risk factors for suicidal ideation and suicide: the role of depression, poor health, and loved ones' messages of support and criticism. Am J Community Psychol 2003; 32: 131-41.

11 Rudd MD, Joiner T, Rajab MH. Treating Suicidal Behaviour. An Effective, Timelimited Approach. Guilford, 2001.

12 McAuliffe C, Corcoran P, Keeley HS, Arensman E, Bille-Brahe U, De Leo D, Fekete S, Hawton K, Hjelmeland H, Kelleher M, Kerkhof AJ, Lonnqvist J, Michel K, Salander-Renberg E, Schmidtke A, Van Heeringen K, Wasserman D. Problem solving ability and repetition of deliberate self-harm: a multicentre study. Psychol Med 2006; 36: 45-55.

13 Townsend E, Hawton K, Altman DG, Arensman E, Gunnell D, Hazell P, House $A$, Van Heeringen $K$. The efficacy of problem solving treatments after deliberate self-harm: meta-analysis of randomized controlled trials with respect to depression, hopelessness and improvement in problems. Psychol Med 2001; 31: 979-88.

14 Comtois KA, Linehan MM. Psychosocial treatments of suicidal behaviours: a practice friendly review. J Clin Psychol 2006; 62: 161-70.

15 Tyrer $P$, Thompson S, Schmidt U, Jones M, Knapp M, Davidson K, Catalan J, Airlie J, Baxter S, Byford S, Byrne G, Cameron S, Caplan R, Cooper S, Ferguson B, Freeman C, Frost S, Godley J, Greenshields J, Henderson J, Holden N, Keech P, Kim L, Logan K, Manley C, MacLeod A, Murphy R, Patience L, Ramsay L, De Munroz S, Scott J, Seivewright H, Sivakumar K, Tata $\mathrm{P}$, Thornton S, Ukoumunne OC, Wessely S. Randomized controlled trial of brief cognitive behaviour therapy versus treatment as usual in recurrent deliberate self-harm: the POPMACT study. Psychol Med 2003; 33: 969-76.

16 Brown GK, Ten Have T, Henriques GR, Xie SX, Hollander JE, Beck AT. Cognitive therapy for the prevention of suicide attempts. A randomized controlled trial. JAMA 2005; 294: 563-70.

17 Linehan MM, Comtois KA, Murray AM, Brown MZ, Gallop RJ, Heard HL, Korslund KE, Tutek DA, Reynolds SK, Lindenboim N. Two-year randomized controlled trial and follow-up of dialectical behavior therapy vs therapy by experts for suicidal behaviors and borderline personality disorder. Arch Gen Psychiatry 2006; 63: 757-66.

18 Giesen-Bloo J, van Dyck R, Spinhoven Ph, van Tilburg W, Dirksen C, van Asselt T, Kremers I, Nadort M, Arntz A. Outpatient psychotherapy for borderline personality disorder: a randomized trial of schema-focused therapy versus transference-focused psychotherapy. Arch Gen Psychiatry 2006; 63: 649-58.

19 Hawton $\mathrm{K}$, Arensman E, Townsend E, Bremner S, Feldman E, Goldney R, Gunnell $D$, Hazell $P$, van Heeringen $K$, House A, Owens D, Sakinofsky I, Traskman-Bendz L. Deliberate self-harm: systematic review of efficacy of psychosocial and pharmacological treatments in preventing repetition. BM 1998; 317: 441-7.

20 Slee N, Arensman E, Garnefski N, Spinhoven P. Cognitive behavioral therapy for deliberate self-harm. Crisis 2007; 28: 175-182.

21 Sheehan DV, Lecrubier Y. Mini-International Psychiatric Interview. M.I.N.I. PLUS. English Version 5.0.0. DSM-IV. University of South Florida, 1992, 1994, 1998. 
22 De Leo D, Bille-Brahe U, Kerkhof A, Schmidtke A. Suicidal Behavior. Hogrefe \& Huber, 2004.

23 Schmidtke A, Bille-Brache U, De Leo D, Kerkhof A. Suicidal Behaviour in Europe. Hogrefe \& Huber, 2004.

24 Beck AT, Steer RA, Brown GK. Manual for the Beck Depression Inventory II. Psychological Corporation, 1996.

25 Van der Does AJW. Handleiding bij de Nederlandse Bewerking van de BDI-II. Swets Test Publishers, 2002.

26 Beck AT, Steer RA, Ball R, Ranieri WF. Comparison of Beck Depression Inventories IA and II in psychiatric outpatients. J Pers 1996; 67: 588-97.

27 Derogatis LR. SCL-90: Administration, Scoring and Procedure Manual-I for the Revised Version. Johns Hopkins University School of Medicine, Clinical Psychometrics Research Unit, 1977.

28 Arindell WA, Ettema JHM. Dutch Translation of the SCL-90. Swets \& Zeitlinger, 1986.

29 Robson, PJ. Development of a new self-report questionnaire to measure selfesteem. Psychol Med 1989; 19: 513-18.

30 Endler NS, Parker JDA. Coping Inventory for Stressful Situations: CISS. Manual. Multi-Health Systems, 1990.

31 Endler NS, Parker JDA. Assessment of multidimensional coping: task, emotion, and avoidance strategies. Psychol Assessment 1994; 6: 50-60.

32 Beck AT, Schuyler D, Herman I. Development of suicidal intent scales. In The Prediction of Suicide (eds AT Beck, HLP Resnik \& DJ Lettieri): 45-8. Charles Press, 1974.

33 Hawton K, Cole D, O'Grady J, Osborn M. Motivational aspects of deliberate self-poisoning in adolescents. Br J Psychiatry 1982; 141: 286-91.

34 Bancroft JH, Skrimshire AM, Simkin S. The reasons people give for taking overdoses. Br J Psychiatry 1976; 128: 538-48.

35 Vliet IM van, Leroy H, Van Megen HJGM. M.I.N.I. PLUS. M.I.N.I. Internationaal Neuropsychiatrisch Interview. Nederlandse Versie 5.0.0. Leids Universitair Medisch Centrum, 2000.

36 Snijders TAB, Bosker RJ. Standard errors and sample sizes in two-level research. J Educational Statist 1993; 18: 237-60.

37 Rasbash J, Steele F, Browne W, Prosser B. A User's Guide to MLwiN, Version 2.0 (Computer Software). University of London, Institute of Education, Centre for Multilevel Modelling, 2004.
38 Thalheimer W, Cook S. How to calculate effect sizes from published research articles: a simplified methodology. Work Learning Research, 2002. http://work-learning.com/effect_sizes.htm.

39 Hox JJ. Multilevel Analysis: Techniques and Applications. Erlbaum, 2002.

40 Maas CJM, Hox JJ. Robustness issues in multilevel regression analysis. Stat Neerl 2004; 58: 127-37.

41 Cohen J. A power primer. Psychol Bull 1992; 112: 155-9.

42 Van Heeringen $\mathrm{K}$ (ed). Understanding Suicidal Behaviour. The Suicidal Process Approach to Research, Treatment and Prevention. Wiley, 2001.

43 Raj MAJ, Kumaraiah V, Bhide AV. Cognitive-behavioural intervention in deliberate self-harm. Acta Psychiatr Scand 2001; 104: 340-5.

44 Nezu A, Nezu C, Perri M. Problem-solving Therapy For Depression: Theory, Research, and Clinical Guidelines. Wiley, 1989.

45 Harriss L, Hawton $\mathrm{K}$. Suicidal intent in deliberate self-harm and the risk of suicide: the predictive power of the Suicide Intent Scale. J Affect Disord 2005; 86: 225-33.

46 Kraemer HC, Wilson T, Fairburn CG, Agras WS. Mediators and moderators of treatment effects in randomized clinical trials. Arch Gen Psychiatry 2002; 59: 877-83

47 Berk MS, Henriques GR, Warman DM, Brown GK, Beck AT. A cognitive therapy intervention for suicide attempters: an overview of the treatment and case examples. Cogn Behav Pract 2004; 11: 265-77.

48 Tyrer $\mathrm{P}$, Tom B, Byford S, Schmidt U, Jones V, Davidson K, Knapp M, MacLeod A, Catalan J; POPMACT Group. Differential effects of manual assisted cognitive behavior therapy in the treatment of recurrent deliberate self-harm and personality disturbance: the POPMACT study. J Personal Disord 2004; 18: $102-16$.

49 Arntz A, Dietzel R, Dreesen L. Assumptions in borderline personality disorder: specificity, stability and relationship with etiological factors. Behav Res Ther 1999; 37: 545-57.

50 Young JE. Cognitive Therapy for Personality Disorders: A Schema-Focused Approach. Professional Resource Press, 1994.

51 Bateman AW, Fonagy P. Psychotherapy for Borderline Personality Disorder: Mentalization Based Treatment. Oxford University Press, 2004. 